\title{
Cytokines as an Adjuvant to Tumor Vaccines: Efficacy of Local Methods of Delivery
}

\author{
Shuji Kurane, MD, Marjorie T. Arca, MD, Atsushi Aruga, MD, PhD, Robert A. Krinock, BS, \\ John C. Krauss, MD, and Alfred E. Chang, MD
}

Background: We examined alternative methods of delivering cytokines as an adjunct for priming lymph node ( $\mathrm{LN}$ ) cells draining sites of vaccine inoculation for the purpose of generating immune cells for adoptive immunotherapy.

Methods: Using syngeneic murine tumors we examined the ability of IL-2, IL-4, or GM-CSF delivered locally to a site of tumor inoculum to induce antitumor reactive draining $\mathrm{LN}$ cells. Mice were inoculated subcutaneously with tumor cells transduced to secrete cytokine; tumor cells admixed with fibroblasts transduced to secrete cytokine; or intralesional inoculation of cytokine in established tumor to induce sensitized LN cells capable of mediating tumor regression in adoptive transfer.

Results: Both IL-4 and GM-CSF cytokines were effective in enhancing the antitumor reactivity of vaccine-primed LN cells compared to IL-2, which was ineffective. The local delivery of GM-CSF by autocrine or paracrine secretion of genetically engineered cells, as well as direct intratumoral delivery was capable of upregulating LN sensitization compared to systemic administration, which did not.

Conclusions: The local delivery of GM-CSF as an adjuvant for tumor vaccination can be accomplished by various methods, including direct injection, which avoids the need for gene transfer.

Key Words: Vaccines-IL-4-GM-CSF-Gene therapy_-Adoptive immunotherapy.

Several recent developments have caused a resurgence of interest in the therapeutic potential of tumor vaccines. Foremost among these is our increasing knowledge about the cellular events involved in the induction of host immunity to tumor antigens. This includes the identification of the key role of dendritic cells necessary to process and present antigen (1-3), the requirements for co-stimulatory molecules to engage $T$ cells (4-6), and the regulatory role of cytokines, which have been classified into type 1 versus type 2 effects (7). A second development is the identification of several human tumorassociated antigens that can serve as targets for the immune system. Many of these tumor-associated antigens

Received February 18, 1997; accepted May 12, 1997.

From the Division of Surgical Oncology (S.K., M.T.A., A.A., R.A.K., A.E.C.), University of Michigan, Ann Arbor, Michigan, and The Cleveland Clinic Foundation (J.C.K.), Cleveland, Ohio, USA.

Address correspondence and reprint requests to Dr. A.E. Chang, University of Michigan Medical Center, 1500 E. Medical Center Dr., Ann Arbor, MI 48109, USA. appear to be normal molecules that are over-expressed by tumor cells, such as HER2/neu in breast, ovarian and lung cancer; CEA in colon cancer; or MAGE 1, MAGE 3 , and tyrosinase in melanoma. Mutated oncogenes (e.g., Ras) or tumor suppressor genes (e.g., p53) associated with carcinogenesis also have been reported to give rise to immune responses against the altered gene products (8). Several of these tumor-associated peptides are now being used as vaccine reagents in clinical studies.

The use of tumor antigens, whether purified peptides or derivatives of whole tumor cells, most likely is more effective if coupled with an immune adjuvant. The earliest observations (by William B. Coley, a surgeon, in the 1890s) that inoculation of viable bacteria into patients' tumors would cause some of them to regress established the foundation that the host immune system could be modulated in favor of an antitumor response. Bacterial products have been the major immune adjuvants used in tumor vaccines for several decades. More recently, the identification of the cytokines as mediators of the im- 
mune system has given investigators more selective reagents for modulating the antitumor response. In particular, the genetic modification of tumor cells to secrete different cytokines has been reported to enhance the immunogenicity of tumor cells in syngeneic murine systems (9) and has formed the rationale for many current gene therapy protocols in cancer patients (10).

In this study, we used various syngeneic murine tumor models to evaluate the efficacy of cytokines IL-2, IL-4, and GM-CSF in upregulating host antitumor immunity. The primary area we examined was the ability of tumor cells and locally delivered cytokines to induce antitumor reactive $T$ cells in tumor-draining lymph nodes (TDLN). We found that local delivery of cytokines (e.g., GMCSF) by several different methods, as opposed to systemic administration, was highly effective in eliciting antitumor reactivity in TDLN. These observations have clinical relevance to the development of tumor vaccines.

\section{MATERIALS AND METHODS}

\section{Mice}

Female C57BL/6J (B6) mice (Jackson Laboratory, Bar Harbor, ME), 6-8 weeks old, were maintained in specific pathogen-free conditions and were used for experiments at age 8 weeks or older.

\section{Tumors}

MCA 205 and MCA 207 are fibrosarcomas induced by 3-methylcholanthrene in $\mathrm{B} 6$ mice. These tumors have been previously characterized as immunologically distinct (11) and also have been identified as weakly immunogenic by virtue of their ability to induce therapeutically effective TIL during tumor growth (11) and their ability to induce pre-effector lymphoid cells in tumordraining lymph nodes $(12,13)$. Another tumor used in this study is the D5 melanoma, a clone of the B16-BL6 melanoma, which was a spontaneous melanoma that arose in a B6 host (14). We have previously characterized the B16-BL6 melanoma as a nonimmunogenic tumor that does not induce pre-effector lymphoid cells in tumor-draining LN (15).

\section{Recombinant retroviruses}

Plasmid DNA subcloning was carried out by standard techniques, using reagents obtained from New England Biolabs (Tozer, MA, USA). All the recombinant viruses have been described previously (15). pMFG-IL-2 was produced by isolating the BamH1 fragment containing the murine IL-2 cDNA, and cloned into the BamH1 site of pMFG. pMFG IL-4 was produced by isolating the BamH1 fragments containing the murine IL-4 cDNA and cloned into the BamH1 site of pMFG. Murine GM-CSF cDNA (a gift from Dr. N. Gough, Melbourne, Australia) was mutated to include an Ncol site at the initiation of ATG and a BamH1 site just $3^{\prime}$ to the termination codon by polymerase chain reaction, then subsequently cloned into the Ncol and BamH1 site of pMFG. Retrovirus vectors were co-transfected with the selectable marker pSV2Neo into $\psi$ crip cells as described (15). Recombinant retrovirus producer cell clones were isolated for the generation of high-titer viruses and used for transduction of tumor cells of cultured fibroblasts. Cytokine-secreting tumor clones or fibroblasts were established by transducing exponentially growing cells with $5 \mathrm{ml}$ of virus supernatants in the presence of polybrene $(8 \mu \mathrm{g} / \mathrm{ml})$, and then subcloned by limiting dilution. The NIH $3 \mathrm{~T} 3$ fibroblast line was obtained from the American Type Culture Collection (ATCC, Rockville, MD) for transduction. Each clone was screened for cytokine production and used in further experiments.

\section{Cytokine assays}

To evaluate IL-2 secretion from the transduced tumor lines, the IL-2-dependent cell line HT-2 (ATCC, Rockville, MD) was used to determine IL-2 concentration as previously described (15). Briefly, $1 \mathrm{ml}$ of medium conditioned by growing $10^{6}$ tumor cells for 24 hours was assayed for cytokine content in a standard 3-[4,5dimethylthiazol-2-yl]-2,5-diphenyltetrazolium bromine (MTT) proliferation assay, with recombinant human IL-2 used as a standard in parallel. To evaluate IL-4 and GMCSF secretion, supernatants were assayed using ELISA kits (Pharmingen, San Diego, CA) with recombinant murine IL-4 and GM-CSF as a known standard run in parallel.

\section{Tumor-draining lymph nodes (TDLN)}

Tumor cells in $0.10 \mathrm{ml}$ Hank's balanced salt solution (HBSS) were inoculated intradermally in the flanks of syngeneic B6 mice. Nine to 14 days later, tumor-draining lymph nodes from each inoculated mouse were harvested under sterile conditions. Lymphocytes were dissociated into single cell suspension by teasing apart the $\mathrm{LN}$ with 25 -gauge needles and then pressing the $\mathrm{LN}$ with the blunt end of a $10-\mathrm{ml}$ plastic syringe.

\section{In vitro anti-CD3/IL-2 activation}

Tumor-draining $L N$ cells were activated for 2 days in vitro with immobilized anti-CD3 antibody (145-2C11 kindly provided by Dr. J. Bluestone, University of Chicago, Chicago, IL) in 24-well tissue culture plates at $4 \times$ $10^{6}$ cells/well. Complete medium (CM) consisted of RPMI 1640 medium supplemented with $10 \%$ heatinactivated fetal calf serum, $0.1 \mathrm{mM}$ nonessential amino 
acids, $1 \mathrm{mM}$ sodium pyruvate, $100 \mathrm{mg} / \mathrm{ml}$ streptomycin, $1000 \mathrm{U} / \mathrm{ml}$ penicillin, $50 \mathrm{mg} / \mathrm{ml}$ gentamicin, $0.5 \mathrm{mg} / \mathrm{ml}$ fungizone (all from GIBCO, Grand Island, NY), and $5 \times$ $10^{5}$ M 2-mercaptoethanol (Sigma, St. Louis, MO). After anti-CD3 activation, the cells were harvested, washed and resuspended at $3 \times 10^{5} \mathrm{cells} / \mathrm{ml}$ in CM containing 60 $\mathrm{IU} / \mathrm{ml}$ of IL-2. Human recombinant IL-2 with a specific activity of 18 million IU/mg (Chiron Therapeutics, Emeryville, CA) was used. The cells were subsequently harvested, washed, and resuspended in HBSS for adoptive immunotherapy.

\section{Adoptive immunotherapy protocol}

B6 mice were injected intravenously with tumor cells in $1 \mathrm{ml}$ of HBSS to establish pulmonary metastases. On day 3, anti-CD3/LL-2 activated LN cells were suspended in $1 \mathrm{ml}$ of HBSS and injected intravenously to each mouse. Some mice were also treated with 60,000 IU IL-2 in $0.5 \mathrm{ml}$ HBSS intraperitoneally twice a day for 4 days to maintain the in vivo proliferation and antitumor reactivity of the transferred cells. Approximately 21 days after tumor inoculation, the mice were sacrificed and the lungs insufflated with a $15 \%$ solution of India ink and bleached by Fekete's solution (16). Sarcoma pulmonary metastases were counted before it was revealed which mice had received which treatment. If mice were found to be inoculated with D5 melanoma, the lungs were insufflated with Fekete's solution only to preserve the tissue for subsequent nodule enumeration. For all experiments, lungs with pulmonary nodules too numerous to count were arbitrarily assigned a value of $>250$ for statistical analysis.

\section{Statistical analysis}

The significance of differences in the numbers of pulmonary metastases between groups was determined by Wilcoxon rank-sum test. Two-sided $p$ values of $<0.05$ were considered statistically significant. No animals were excluded from the statistical analysis.

\section{RESULTS}

\section{Immune responses induced by tumors genetically modified to secrete IL-2, IL-4, or GM-CSF}

Using the MCA 205 tumor, we produced stable clones by retroviral transduction that secreted $\mathrm{IL}-2, \mathrm{IL}-4$, or GM-CSF. The wild-type MCA 205 did not secrete any of these cytokines constitutively. IL-2-secreting clones named IL-2 (high) and IL-2 (low) secreted 140 and 25 $\mathrm{u} / 10^{6}$ cells $/ 24 \mathrm{~h}$, respectively. IL-4 secreting clones termed IL-4 (high) and IL-4 (low) secreted 200 and 16 $\mathrm{u} / 10^{6}$ cells $/ 24 \mathrm{~h}$, respectively. A GM-CSF-secreting clone produced $180 \mathrm{ng} / 10^{6}$ cells $/ 24 \mathrm{~h}$ of transgenic protein. The tumorigenicity of these clones was assessed by inoculating $3 \times 10^{6}$ tumor cells intradermally in normal syngeneic B6 hosts (5 animals/group) and tumor growth measured in perpendicular dimensions (Fig. 1). Both IL-2 secreting clones were rejected, with the IL-2 (high) being rejected earlier. All of these animals developed systemic immunity to MCA 205 by their ability to reject a subsequent challenge of $10^{7}$ viable tumor cells. Both IL-4 secreting clones manifested reduced tumorigenicity; this was greatest with the IL-4 (high) clone. Two of five animals inoculated with the IL-4 (high) tumor cells completely rejected tumor, whereas the remaining animals had barely detectable residual tumor. Mice inoculated with GM-CSF-secreting cells did not demonstrate significantly diminished tumorigenicity for the first 21 days. After 21 days, one of five animals bearing a tumor measuring $100 \mathrm{~mm}^{2}$ went on to completely reject that tumor. The other mice became severely cachectic and went on to die from apparent toxicity as a result of GM-CSF secretion.

Using our adoptive immunotherapy model, we examined the immune reactivity of lymphoid cells derived from $\mathrm{LN}$-draining cytokine-secreting MCA 205 tumors. Tumor-draining lymph nodes (TDLN) were removed approximately 9 to 10 days after intradermal tumor inoculation and activated ex vivo as described in the Methods



FIG. 1. Tumorigenicity of transduced cytokine-secreting MCA 205 tumors. $3 \times 10^{6}$ tumor cells were inoculated intradermally into groups of five mice. Wild-type MCA 205 (WT-205) was used as a control. Tumors were measured twice a week in perpendicular diameter and a tumor area in $\mathrm{mm}^{2}$ was calculated. (A) The growth of tumor lines secreting IL-2. (B) Tumor lines secreting IL-4. (C) The growth of tumor cells secreting GM-CSF. 
section. Activated TDLN cells were assessed for their antitumor reactivity by adoptive transfer into mice bearing micrometastatic pulmonary disease. As summarized in Table 1, the IL-2 (high) clone of MCA 205 did not enhance the antitumor reactivity of TDLN cells when compared to the wild-type tumor. However, in a similar series of experiments, we found that the IL-4 (high) clone of MCA 205 did augment antitumor reactivity of TDLN cells in two of two experiments (Table 2). Secretion of GM-CSF by MCA 205 tumor cells also enhanced antitumor reactivity in TDLN cells (Table 3).

To further explore the adjuvant effect of GM-CSF, we developed several cytokine-secreting D5 clones. These D5 clones secreted 400,150 , and $75 \mathrm{ng} / 10^{6}$ cells $/ 24 \mathrm{~h}$ of transgenic protein. Compared with the wild-type tumor, these clones did not have significantly altered tumorigenicity (data not shown). In the adoptive immunotherapy model, the GM-CSF-secreting tumor clones were found to be effective in the induction of antitumor reactivity in TDLN, whereas the wild-type D5 tumor did not (Table 4, Exp. 1). The amount of GM-CSF secretion was found to correlate with the induction of antitumor reactivity elicited in the TDLN (Table 4, Exp. 3).

\section{Local delivery of GM-CSF by transduced fibroblasts}

We proceeded to examine whether tumor cells were required to secrete GM-CSF to observe an immune adjuvant effect or whether it can be delivered locally by another cell. 3T3 fibroblasts were retrovirally transduced to secrete GM-CSF. A stable clone that secreted $50 \mathrm{ng} /$ $10^{6}$ cells $/ 24 \mathrm{~h}$ was identified and used for further experiments. Tumor cells (D5 or MCA 207) were mixed with

TABLE 1. Treatment of established MCA 205 lung metastases with effector cells generated from $L N$-draining wild-type or IL-2 (high)-secreting MCA 205

\begin{tabular}{lccc}
\hline & $\begin{array}{c}\text { No. of } \\
\text { cells } \times 10^{6}\end{array}$ & \multicolumn{2}{c}{$\begin{array}{c}\text { Mean no. of pulmonary } \\
\text { metastases (SEM) }\end{array}$} \\
\cline { 3 - 4 } TDLN cells $^{\mathrm{a}}$ & transferred $^{\mathrm{b}}$ & Exp. 1 & Exp. 2 \\
\hline None & None & $>250$ & $>250$ \\
Wild-type & 6 & $231(14)$ & $118(41)^{\mathrm{c}}$ \\
& 12 & $98(42)^{\mathrm{c}}$ & $71(34)^{\mathrm{c}}$ \\
IL-2 (high) & 6 & $222(18)$ & $>250$ \\
& 12 & $130(37)^{\mathrm{c}}$ & $225(16)$ \\
\hline
\end{tabular}

(SEM), (standard error of measurement).

${ }^{a}$ Mice were inoculated intradermally in the flank with $2-3 \times 10^{6}$ tumor cells. Tumor-draining lymph nodes (TDLN) were harvested and activated in vitro 12 to 14 days later.

${ }^{b}$ TDLN cells were transferred intravenously to the mice 3 days after intravenous MCA 205 tumor inoculation. All mice received $60,000 \mathrm{u}$ of IL-2 intraperitoneally twice a day for 4 days starting on the day of cell transfer.

${ }^{\circ} p<0.05$ compared to mice not treated with TDLN cells.
TABLE 2. Treatment of established MCA 205 lung metastases with effector cells generated from $L N$-draining wild-type or IL-4 (high)-secreting MCA 205

\begin{tabular}{lccc}
\hline \multirow{2}{*}{$\begin{array}{c}\text { Source of } \\
\text { TDLN cells }\end{array}$} & $\begin{array}{c}\text { No. of } \\
\text { cells } \times 10^{\mathrm{a}} \\
\text { transferred }\end{array}$ & \multicolumn{2}{c}{$\begin{array}{c}\text { Mean no. of pulmonary } \\
\text { metastases (SEM) }\end{array}$} \\
\cline { 3 - 4 } None & None & Exp. 1 & Exp. 2 \\
Wild-type & 6 & $153(44)$ & $>250$ \\
& 12 & $91(22)^{\mathrm{b}}$ & $54(15)^{\mathrm{b}}$ \\
IL-4 (high) & 6 & $65(20)^{\mathrm{b}}$ & $61(20)^{\mathrm{b}}$ \\
& 12 & $22(8)^{\mathrm{b}, \mathrm{c}}$ & $17(5)^{\mathrm{b}, \mathrm{c}}$ \\
\end{tabular}

(SEM), (standard error of measurement).

${ }^{a}$ Adoptive immunotherapy protocol is the same as that described in Table 1 . All mice received $60,000 \mathrm{u}$ of IL-2 intraperitoneally twice a day for 4 days starting on the day of cell transfer.

${ }^{\mathrm{b}} p<0.05$ compared to mice with no treatment or treated with $\mathrm{IL}-2$ only.

${ }^{c} p<0.05$ compared to mice treated with the same number of wildtype TDLN cells.

equal numbers of GM-CSF-secreting fibroblasts and inoculated intradermally into mice. Nine days later, TDLN cells were removed, activated ex vivo, and assessed for antitumor reactivity in the adoptive immunotherapy model. As summarized in Table 5, non-transduced 3T3 cells admixed with D5 tumor had no adjuvant effect. However, GM-CSF-secreting 3T3 manifested an adjuvant effect in the induction of tumor reactive TDLN for both D5 and MCA 207 tumors. This demonstrated that the paracrine delivery of GM-CSF was effective in augmenting host cellular immunity against tumor and did not require tumor cells to secrete the cytokine.

\section{Local delivery of GM-CSF by intratumoral inoculation of soluble GM-CSF}

Finally, we examined whether the immune adjuvant effect mediated by GM-CSF could be induced by the

TABLE 3. Treatment of established MCA 205 lung metastases with effector cells generated from $L N$-draining wild-type or GM-CSF-secreting MCA 205

\begin{tabular}{lccc}
\hline \multirow{2}{*}{$\begin{array}{c}\text { Source of } \\
\text { TDLN cells }\end{array}$} & $\begin{array}{c}\text { No. of } \\
\text { cells } \times 10^{\mathrm{a}} \\
\text { transferred }\end{array}$ & \multicolumn{2}{c}{$\begin{array}{c}\text { Mean no. of pulmonary } \\
\text { metastases (SEM) }\end{array}$} \\
\cline { 3 - 4 } None & 0 & Exp. 1 & Exp. 2 \\
Wild-type & 6 & $153(44)$ & $>250$ \\
& 12 & $91(22)^{\mathrm{b}}$ & $146(53)^{\mathrm{b}}$ \\
GM-CSF & 6 & $81(25)^{\mathrm{b}}$ & $27(13)^{\mathrm{b}}$ \\
& 12 & $36(13)^{\mathrm{b}, \mathrm{c}}$ & $0^{\mathrm{b}}$ \\
\hline
\end{tabular}

(SEM), (standard error of measurement).

${ }^{a}$ Adoptive immunotherapy protocol is the same as that described in Table 1. All mice received $60,000 \mathrm{u}$ of IL-2 intraperitoneally twice a day for 4 days starting on the day of cell transfer.

${ }^{\mathrm{b}} p<0.05$ compared to mice not treated with TDLN cells.

${ }^{\circ} p<0.05$ compared to mice treated with the same number of wildtype TDLN cells. 
TABLE 4. Treatment of established D5 lung metastases with effector cells from $L N$-draining wild-type or $G M$-CSF-secreting D5

\begin{tabular}{lcccc}
\hline & & \multicolumn{3}{c}{$\begin{array}{c}\text { Mean no. of pulmonary } \\
\text { metastases (SEM) }\end{array}$} \\
\cline { 3 - 5 } $\begin{array}{l}\text { Source of } \\
\text { TDLN cells }\end{array}$ & $\begin{array}{c}\text { Amount of } \\
\text { GM-CSF }^{b}\end{array}$ & Exp. 1 & Exp. 2 & Exp. 3 \\
\hline None & - & $>250$ & $>250$ & $>250$ \\
None & - & $>250$ & $>250$ & $>250$ \\
Wild-type & 0 & $>250$ & ND & ND \\
D5G6 & 400 & $91(28)^{\mathrm{c}}$ & $7(11)^{\mathrm{c}}$ & $29(26)^{\mathrm{c}}$ \\
D5G7 & 150 & ND & ND & $43(21)^{\mathrm{c}}$ \\
D5G5 & 75 & ND & ND & $74(28)^{\mathbf{c}, \mathbf{d}}$ \\
\hline
\end{tabular}

(SEM), (standard error of measurement).

${ }^{a}$ Mice were inoculated intradermally in the flank with $10^{6}$ tumor cells. Nine days later, TDLN were harvested and activated in vitro. Seven $\times 10^{7}$ TDLN cells were administered intravenously in mice bearing 4-day established lung metastases. All mice received 60,000 u $\mathrm{IL}-2$ intraperitoneally twice daily for 4 days starting on the day of cell transfer.

${ }^{\mathrm{b}} \mathrm{GM}-\mathrm{CSF}$ secretion by tumor cells in $\mathrm{ng} / 10^{6}$ cells $/ 24 \mathrm{~h}$.

${ }^{c} p<0.05$ compared to mice not treated with TDLN cells.

d $p<0.05$ compared to mice treated with D5G6 TDLN cells.

intratumoral inoculation of cytokine. This would obviate the need to develop genetically modified tumor cells or fibroblasts. To simulate amounts of GM-CSF comparable to a growing inoculum of viable $10^{6}$ D5G6 tumor cells that secrete $400 \mathrm{ng} / 24 \mathrm{~h}$, soluble cytokine was injected intratumorally twice daily at increasing doses starting on day 1 after intradermal inoculation of $10^{6}$ wild-type D5 tumor cells. The total amount of GM-CSF delivered intratumorally per day was: $400 \mathrm{ng}$ (days 1 and 2); $800 \mathrm{ng}$ (days 3 and 4); $1600 \mathrm{ng}$ (days 5 and 6); and $3200 \mathrm{ng}$ (days 7 and 8). TDLN were harvested on day 9

TABLE 5. Treatment of established D5 or MCA 205 lung metastases with effector cells generated from $L N$ primed by tumor admixed with GM-CSF secreting fibroblasts

\begin{tabular}{lcc}
\hline & \multicolumn{2}{c}{$\begin{array}{c}\text { Mean no. of pulmonary } \\
\text { metastases (SEM) }\end{array}$} \\
\cline { 2 - 3 } \multicolumn{1}{c}{ Source of TDLN cells } & D5 (Exp. 1) & MCA 207 (Exp. 2) \\
\hline A. None & $232(8)$ & $>250$ \\
B. Tumor & $152(25)$ & 214 \\
C. Tumor + 3T3 & $176(32)$ & ND \\
D. Tumor + 3T3/GM-CSF & $44(9)^{\mathrm{c}}$ & $98^{\mathrm{d}}$ \\
\hline
\end{tabular}

(SEM), (standard error of measurement).

${ }^{a}$ Mice were inoculated intradermally in the flank with either $10^{6} \mathrm{D} 5$ (Exp. 1) or $1.5 \times 10^{6} \mathrm{MCA} 207$ (Exp. 2) tumor cells with or without the same number of $3 \mathrm{~T} 3$ fibroblasts or GM-CSF-secreting fibroblasts (3T3/GM-CSF). Nine days later, TDLN were harvested and activated in vitro.

${ }^{\mathrm{b}}$ Mice bearing 3- to 5-day established lung metastases received $2 \times$ $10^{7}$ activated TDLN cells intravenously. All mice received $60,000 \mathrm{u}$ of IL-2 intraperitoneally twice a day for 4 days starting on the day of cell transfer.

${ }^{c} p<0.01$ compared to groups $\mathrm{A}, \mathrm{B}$, and $\mathrm{C}$.

${ }^{\mathrm{d}} p<0.001$ compared to groups $\mathrm{A}$ and $\mathrm{B}$. and assessed for antitumor reactivity in the adoptive immunotherapy model. As shown in Table 6, intratumoral inoculation of GM-CSF resulted in the induction of tumor-reactive TDLN that was comparable to the TDLN induced by GM-CSF-secreting D5G6 cells. To determine whether systemic administration of GM-CSF can have the same adjuvant effect as intratumoral delivery, the same doses and schedule of cytokine were administered intraperitoneally in mice inoculated with wild-type D5 tumor. As summarized in Table 6, the intraperitoneal administration of GM-CSF was not an effective delivery route to induce tumor reactive TDLN cells. In this latter experiment, D5G6 tumors were successful in the priming of sensitized TDLN cells and served as a positive control.

\section{DISCUSSION}

Using the weakly immunogenic MCA 205 sarcoma, we found that transduced tumor cells that elaborated IL-4 or GM-CSF, but not IL-2, resulted in enhanced sensitization of TDLN cells reactive to tumor. This extends our previous observations made with the B16-BL6 tumor, a nonimmunogenic tumor (17). Both of these cytokines have been reported to be important in the maturation and growth of dendritic cells in vitro (18). Dendritic cells are potent antigen-presenting cells capable of processing tumor antigen and sensitizing naive $T$ cells in both in vitro and in vivo models $(1-3)$. We hypothesize that the adjuvant effect of IL-4 or GM-CSF in this model involves the recruitment or stimulation of dendritic cells to the local site of tumor antigen to facilitate the afferent arm of the immune response. As a helper type 1 cytokine, IL-2

TABLE 6. Efficacy of systemic versus intratumoral administration of GM-CSF to prime D5-draining lymph nodes

\begin{tabular}{lcc}
\hline & \multicolumn{2}{c}{ Mean no. of pulmonary metastases (SEM) } \\
\cline { 2 - 3 } Source of cells & Exp. 1 & Exp. 2 \\
\hline A. None & $>250$ & $>250$ \\
B. D5 & $>250$ & ND \\
C. D5 + i.p. GM-CSF & $>250$ & ND \\
D. D5 + i.t. GM-CSF & ND & $2(2)^{\mathrm{d}}$ \\
E. D5G6 & $148(28)^{\mathrm{c}}$ & $0^{\mathrm{d}}$ \\
\hline
\end{tabular}

(SEM), (standard error of measurement).

${ }^{a}$ Mice were inoculated intradermally in the flank with $10^{6} \mathrm{D} 5$ tumor cells. GM-CSF was administered intraperitoneally or intratumorally as described in the Results section. TDLN were harvested 9 days later and activated in vitro.

${ }^{\mathrm{b}}$ Mice bearing 4-day established lung metastases received 6-7 $\times 10^{7}$ activated TDLN. All mice received 60,000 u of IL-2 intraperitoneally twice a day for 4 days starting on the day of cell transfer.

${ }^{c} p<0.05$ compared to groups A, B, and C.

${ }^{\mathrm{d}} p<0.001$ compared to group $\mathrm{A}$. 
acts mainly to promote expansion of antigen-activated $T$ cells in the efferent arm of the immune response, and hence did not augment the induction of immune TDLN cells on a per cell basis.

Many gene therapy trials in cancer patients are investigating the potential of tumor cells genetically modified to secrete cytokines as vaccines for active specific immunotherapy in the advanced disease setting in phase I trials (10). The likelihood of therapeutic benefit seems minimal in this clinical setting. These vaccines ultimately may prove more useful in the postoperative setting after patients' primary tumors have been resected and they are at risk for micrometastatic disease. Based on our animal models, in which we can successfully treat advanced, macroscopic disease, we are currently evaluating GM-CSF-secreting autologous tumor cells as a vaccine to prime draining $\mathrm{LN}$ cells for adoptive immunotherapy in patients with stage IV melanoma (19). As per the animal model, this requires the retrieval of tumor tissue and ex vivo genetic engineering of tumor cells to express GM-CSF. There can be technical difficulties in the culture of tumor cells and successful gene transfer to achieve adequate cytokine production; therefore, we and others have looked at alternate methods to deliver cytokines to the microenvironment of tumor cells.

Fibroblasts are readily cultured from a small sample of skin. In our experience, we can establish a fibroblast line from human skin in 2 weeks. In this report, we demonstrated that allogeneic fibroblasts transduced to secrete GM-CSF can be admixed with tumor cells to prime draining LN cells. This extends observations made by other investigators that cytokine-secreting fibroblasts admixed with tumor cells result in altered tumorigenicity $(20,21)$. The use of fibroblasts that secrete IL-4 admixed with autologous tumor is currently being examined in a vaccine trial in melanoma patients (22).

Another delivery method that we found effective was the direct intratumoral inoculation of soluble GM-CSF. This approach resulted in comparable priming of TDLN cells compared with gene-modified tumor cells. By contrast, the systemic administration of an equivalent dose and schedule of GM-CSF was not effective. This underscores the importance of a relatively high concentration of the cytokine in the tumor cell microenvironment to stimulate an immune response. In a rat model, the coadministration of soluble GM-CSF and antigenic peptide intradermally was more effective than complete Freund's adjuvant mixed with peptide in the induction of antipeptide immunity (23). The use of soluble GM-CSF is currently being investigated as an adjuvant for autologous tumor cell vaccinations in patients with stage IV melanoma. This therapy has resulted in tumor regression in a subgroup of patients (24).

The ability to transfer genes into somatic cells in vivo via a variety of different approaches offers opportunities to modify established tumors to express cytokines. The use of viruses as vectors engineered to introduce cytokine genes into tumors in vivo has been reported to be highly efficient at gene transfer. Viruses that are effective for in vivo gene transfer are adenoviruses and the pox viruses (e.g., vaccinia) (25). The application of vaccinia virus that contains the gene for GM-CSF is being tested as a method for direct intratumoral inoculation in patients with melanoma and bladder cancer $(26,27)$. A potential impediment to using viral vectors repeatedly in the same patient is the development of immunity against immunogenic viral epitopes expressed by the vector, which may render them less effective. Alternate forms of localized in vivo gene transfer that avoid viral vectors involve the use of nonviral plasmids encoding the gene of interest. Plasmids complexed with liposomes can be directly injected into established tumors to accomplish successful gene transfer into tumor cells. We have used this approach in both animal and human tumor systems to stimulate an effective antitumor immune response using a foreign major histocompatibility complex class I gene $(28,29)$. Another method of transferring plasmids in vivo is with the use of a "gene gun." Plasmids attached to microgold particles are bombarded onto the tissue target to mediate gene transfer. This technique has been used in animal models to transfer cytokine genes into established tumors with successful tumor regression (30). This technique is currently being tested with cytokine genes in cancer clinical trials (N.S. Yang, personal communication).

In summary, both IL-4 and GM-CSF appear to be effective cytokines as adjuvants in tumor vaccines. The adjuvant effect depends on the level of cytokine available in the local microenvironment of the vaccine. Several different methods of delivering cytokine to the site of the vaccine are effective, including local inoculation of soluble cytokine.

Acknowledgements: This study was supported in part by NIH grant PO1 CA59327 (A.E.C.) and the Gillson Longenbaugh Foundation. The authors wish to acknowledge the excellent secretarial assistance of Ms. Susan Sackrider in compiling this manuscript.

\section{REFERENCES}

1. Hsu FJ, Benike C, Fagnoni F, Liles TM, Czerwinski D, Taidi B, Engleman EG, Levy R. Vaccination of patients with B-cell lymphoma using autologous antigen-pulsed dendritic cells. Nature Medicine 1996; 2(1):52-8. 
2. Mayordomo JI, Zorina T, Storkus WJ, et al. Bone marrow-derived dendritic cells pulsed with synthetic tumour peptides elicit protective and therapeutic antitumour immunity. Nature Medicine 1995; 1(12):1-6.

3. Reeves ME, Royal RE, Lam JS, Rosenberg SA, Hwu P. Retroviral transduction of human dendritic cells with a tumor-associated antigen gene. Cancer Res 1996;56:5672-7.

4. Huang AYC, Bruce AT, Pardoll DM, Levitsky HI. Does B7-1 expression confer antigen-presenting cell capacity to tumors in vivo? J Exp Med 1996;183:769-76.

5. Baskar S, Clements VK, Glimcher LH, Nabavi N, OstrandRosenberg S. Rejection of MHC class II-transfected tumor cells requires induction of tumor-encoded B7-1 and/or B7-2 costimulatory molecules. J Immunol 1996;156:3821-7.

6. Yang G, Mizuno MT, Hellström KE, Chen L. B7-negative versus B7-positive P815 tumor. J Immunol 1997;158:851-8.

7. Mosmann TR, Sad S. The expanding universe of T-cell subsets: Th1, Th2 2 and more. Immunol Today 1996:17:138-46.

8. Cheever MA, Disis ML, Bernhard $\mathrm{H}$, et al. Immunity to oncogenic proteins. Immunol Rev 1995;145:33-59.

9. Miller AR, McBride WH, Hunt K, Economou JS. Cytokinemediated gene therapy for cancer. Ann Surg Oncol 1994;1:436-50.

10. Roth IA, Cristiano RJ. Gene therapy for cancer: what have we done and where are we going? J Natl Cancer Inst 1997;89:21-39.

11. Barth RJ, Bock SN, Mulé JJ, Rosenberg SA. Unique murine tumor-associated antigens identified by tumor infiltrating lymphocytes. J Immunol 1990;144:1531-7.

12. Yoshizawa H, Chang AE, Shu S. Specific adoptive immunotherapy mediated by tumor-draining lymph node cells sequentially activated with anti-CD3 and IL-2. J Immunol 1991;147:729-37.

13. Aruga A, Aruga E, Cameron MJ, Chang AE. Different cytokine profiles released by $\mathrm{CD}^{+}$and $\mathrm{CD} 8^{+}$tumor-draining lymph node cells involved in mediating tumor regression. J Leukoc Biol 1997; 61:1-10.

14. Arca MJ, Krauss JC, Aruga A, Cameron MJ, Shu S, Chang AE. Therapeutic efficacy of $\mathrm{T}$ cells derived from lymph nodes draining a poorly immunogenic turnor transduced to secrete granulocytemacrophage colony-stimulating factor. Cancer Gene Ther 1996;3: $39-47$.

15. Geiger JD, Wagner PD, Cameron MJ, Shu S, Chang AE. Generation of T-cells reactive to the poorly immunogenic B16-BL6 melanoma with efficacy in the treatment of spontaneous metastases. $J$ Immunother 1993;13:153-65.

16. Wexler $\mathrm{H}$. Accurate identification of experimental pulmonary metastases. J Natl Cancer Inst 1966;36:641-5.

17. Arca MJ, Krauss JC, Strome SE, Cameron MJ, Chang AE. Diverse manifestations of tumorigenicity and immunogenicity displayed by the poorly immunogenic B16-BL6 melanoma transduced with cytokine genes. Cancer Immunol Immunother 1996;42:237-45.

18. Sallusto F, Lanzavecchia A. Efficient presentation of soluble an- tigen by cultured human dendritic cells is maintained by granulocyte/macrophage colony-stimulating factor plus interleukin 4 and downregulated by tumor necrosis factor a. J Exp Med 1994;179: 1109-18.

19. Chang AE, Sondak VK, Bishop DK, Nickoloff BJ, Mulligan RC, Mulé JJ. Adoptive immunotherapy of cancer with activated lymph node cells primed in vivo with autologous tumor cells transduced with the GM-CSF gene. Hum Gene Ther 1996:7:773-92.

20. Shawler DL, Dorigo O, Gjerset RA, Royston I, Sobo RE, Fakhrai $\mathrm{H}$. Comparison of gene therapy with interleukin-2 gene modified fibroblasts and tumor cells in the murine CT-26 model of colorectal carcinoma. J Immunother 1995;17:201-8.

21. Tahara H, Zeh HJ, Storkus WJ, et al. Fibroblasts genetically engineered to secrete interleukin 12 can suppress tumor growth and induce antitumor immunity to a murine melanoma in vivo. Cancer Res 1994;54:182-9.

22. Lotze MT, Rubin JT, Carty S, et al. Gene therapy of cancer: a pilot study of IL-4 gene-modified fibroblasts admixed with autologous tumor to elicit an immune response. Hum Gene Ther 1994;5:4155.

23. Disis ML, Bernhard H, Shiota FM, Hand SL, Gralow JR, Huseby ES, Gillis S, Cheever MA. Granulocyte-macrophage colonystimulating factor: an effective adjuvant for protein and peptidebased vaccines. Blood 1996;88:202-10.

24. Leong SPL, Enders-Zohr P, Zhou YM, Allen RE, Sagebiel RW, Glassberg AB, Hayes FA. Active specific immunotherapy with GM-CSF as an adjuvant to autologous melanoma (AM) vaccine in metastatic melanoma. Proc ASCO 1996;15:437.

25. Meko JB, Yin JH, Tsung K, Norton JA. High cytokine production and effective antitumor activity of a recombinant vaccinia virus encoding murine interleukin 12. Cancer Res 1995;55:4765-70.

26. Lee SS, Eisenlohr LC, McCue PA, Mastrangelo MJ, Lattime EC. Intravesical gene therapy: in vivo gene transfer using recombinant vaccinia virus vectors. Cancer Res 1994;54:3325-8

27. Lattime EC, Lee SS, Eisenlohr LC, Mastrangelo MJ. In situ cytokine gene transfection using vaccinia virus vectors. Semin Oncol 1996;23:88-100.

28. Nabel GJ, Nabel EG, Yang Z-Y, et al. Direct gene transfer with DNA-liposome complexes in melanoma: expression, biologic activity, and lack of toxicity in humans. Proc Natl Acad Sci USA 1993;90:11307-11.

29. Nabel GJ, Gordon D, Bishop DK, et al. Immune response in human melanoma after transfer of an allogeneic class I major histocompatibility complex gene with DNA-liposome complexes. Proc Natl Acad Sci USA 1996;93:15388-93.

30. Rakhmilevich AL, Turner J, Ford MJ, McCabe D, Sun WH, Sondel PM, Grota K, Yang N-S. Gene gun-mediated skin transfection with interleukin 12 gene results in regression of established primary and metastatic murine tumors. Proc Natl Acad Sci USA 1996; 93:6291-6. 